\title{
Antimicrobial resistance profile of Salmonella present in organic farming in Selangor, Malaysia
}

\author{
${ }^{1}$ Thung, T.Y., ${ }^{2, *}$ Mazlan, N., ${ }^{3}$ Lee, E., ${ }^{4}$ New, C.Y., ${ }^{1}$ Tan, C.W., ${ }^{1}$ Son, R., ${ }^{5}$ Rinai, K.R., \\ ${ }^{6}$ Anua, S.M. and ${ }^{7,8}$ Mastor, N.N. \\ ${ }^{1}$ Department of Food Science, Faculty of Food Science and Technology, Universiti Putra Malaysia, 43400 \\ UPM Serdang, Selangor, Malaysia \\ ${ }^{2}$ Department of Diagnostic and Allied Science, Faculty of Health and Life Sciences, Management and \\ Science University, 40100 Shah Alam, Selangor, Malaysia \\ ${ }^{3}$ Food Safety and Food Integrity, Institute of Tropical Agriculture and Food Security (ITAFoS), Universiti \\ Putra Malaysia, 43400 Serdang, Selangor, Malaysia \\ ${ }^{4}$ Go Plus Services Sdn. Bhd., 97A, Jalan BP 6/3, Bandar Bukit Puchong, 47120 Puchong, Selangor, \\ Malaysia \\ ${ }^{5}$ Institute of Bioscience, Universiti Putra, Malaysia, 43400 UPM Serdang, Selangor, Malaysia \\ ${ }^{6}$ Environmental and Occupational Health Program, School of Health Sciences, Health Campus Universiti \\ Sains Malaysia, 16150 Kubang Kerian, Kelantan, Malaysia \\ ${ }^{7}$ Biotechnology Research Institute, University Malaysia Sabah, Jalan UMS, 88400 \\ Kota Kinabalu, Sabah, Malaysia \\ ${ }^{8}$ Faculty of Medicine and Health Sciences, University Malaysia Sabah, Jalan UMS, 88400 Kota Kinabalu, \\ Sabah, Malaysia.
}

\section{Article history:}

Received: 22 June 2020

Received in revised form: 16 July 2020

Accepted: 9 August 2020

Available Online: 26

September 2020

Keywords:

Antimicrobial resistance,

Salmonella,

Organic farming,

Vegetables

DOI:

https://doi.org/10.26656/fr.2017.4(6).288

\begin{abstract}
Presence of Salmonella in organic farming may lead to contamination in fresh produce. This study was designed to detect Salmonella contamination in organic vegetable farm situated in Serdang and to evaluate the antibiotic susceptibility profiles of the isolates. A total of 460 samples of brinjal, cucumber, ladies' fingers and soil were collected and examined for the presence of Salmonella. The obtained isolates were identified and confirmed by biochemical characterization and serotyping. Antibiotic sensitivity profiles of the isolates were determined by using agar disk diffusion method. Salmonella spp. was detected in brinjal $(1.7 \%, \mathrm{n}=2)$, cucumber $(1.7 \%, \mathrm{n}=2)$, ladies' fingers $(2.5 \%, \mathrm{n}=3)$ and soil $(5.0 \%, \mathrm{n}=5)$ samples. The prevalent serovars were Salmonella enterica ser. Senftenberg, S. enterica ser. Weltevreden and S. enterica ser. Corvallis. All strains were resistance to penicillin and vancomycin, with multiple antibiotic resistance (MAR) index between 0.21 and 0.36, demonstrated here as multi-drug resistant (MDR) Salmonella. The result highlighted that organic vegetables constituted potential sources of Salmonella, informing continuous monitoring and tightened surveillance are necessary to ensure food safety.
\end{abstract}

\section{Introduction}

Organic products especially vegetables and fruits are most consumed by all races (Malays, Chinese and Indians) in Malaysia due to free chemicals, all-natural and good for health. Despite the health benefits, the risk of microbiological contamination on fresh organic produce is concerning. Generally, fresh produce might be contaminated by foodborne pathogens which present in the soil or introduced with organic fertilizers, as well as irrigation (Kuan et al., 2017). Colonization of foodborne pathogens frequently occurs during cultivation (from soil), harvesting, processing and transport. Previous studies report that raw vegetables are contaminated by foodborne pathogens like Salmonella spp. at the retail level, either wet markets or hypermarkets (Kuan et al., 2017; Abatcha et al., 2018; Saw et al., 2020).

Salmonellosis is caused by ingesting Salmonella spp. People infected with Salmonella may develop diarrhoea, fever and abdominal cramps within 12 to 72 hours after 
infection which could last up to seven days (Saira Banu et al., 2019; FDA, 2020). More severe cases could become fatal in which it was estimated that approximately 450 mortalities each year in the United States due to acute salmonellosis (FDA, 2020). A wide variety of antimicrobial drugs are employed to treat Salmonella infections. However, the emergence and spread of antimicrobial-resistant Salmonella isolates constitute a global challenge for the effective treatment and control of these infections (Hur et al., 2012). In particular, multidrug-resistant (MDR) Salmonella has been reported in retail vegetables (post-harvest level) either collected from organic farms or traditional farms (Abakpa et al., 2015; Wadamori et al., 2016). An earlier study found that most isolated MDR Salmonella strains such as $S$. enterica ser. Albany, $S$. enterica ser. Brancaster, S. enterica ser. Corvallis, S. enterica ser. Dusseldorf and $S$. enterica ser. Paratyphi B. are widely distributed in vegetables at retail level (Abatcha et al., 2018). Therefore, it is vital to strengthen or enhanced surveillance typically at the pre-harvest level, which is critical in the current era of rapidly increasing antimicrobial resistance. This is the first study to detect Salmonella spp. contamination in the organic vegetable farm (pre-harvest level) in Serdang and to evaluate antibiotic resistance patterns in the region.

\section{Materials and methods}

\subsection{Sample collection}

In this study, 120 organic vegetable samples each of brinjal, cucumber, ladies' fingers and 100 soil samples (total 460) were collected utilizing systematic sampling method repeated for every two to three times per week from April 2018 to May 2019 at TPKM4 organic farm, Serdang (Figure 1) located near to the Universiti Putra Malaysia. All samples were brought aseptically to the laboratory for Salmonella isolation.

\subsection{Isolation and identification of Salmonella}

For isolation of Salmonella from vegetable and soil, $25 \mathrm{~g}$ and $10 \mathrm{~g}$ of samples were pre-enriched in $225 \mathrm{~mL}$ and $90 \mathrm{~mL}$ of buffered peptone water (BPW) (Merck, Darmstadt, Germany), respectively, followed by incubation at $37^{\circ} \mathrm{C}$ for $24 \mathrm{hrs}$. After pre-enrichment, 0.1 $\mathrm{mL}$ suspensions were added to $9.9 \mathrm{~mL}$ of Rappaport Vasiliadis Soy (RVS) broth (Merck, Darmstadt, Germany) and incubated at $37^{\circ} \mathrm{C}$ for $24 \mathrm{hrs}$. Then, the enriched samples were plated on Xylose Lysine Deoxycholate (XLD) agar and incubated at $37^{\circ} \mathrm{C}$ for 24 hrs. Presumptive colonies in XLD plates were selected and subjected to biochemical characterization (decarboxylation of lysine, hydrolysis of urea, indole test) as recommended by ISO 6579:2002 (ISO, 2002). Serotyping of Salmonella isolates was carried out at Salmonella reference centre at Public Health Laboratory, Ipoh, Malaysia in accordance with the Kauffmann-White scheme.

\subsection{Antibiotic susceptibility test}

Antibiotic susceptibility was evaluated using disc diffusion method on the following 14 antimicrobial agents: amoxicillin $(30 \mu \mathrm{g})$, ampicillin $(10 \mu \mathrm{g})$,
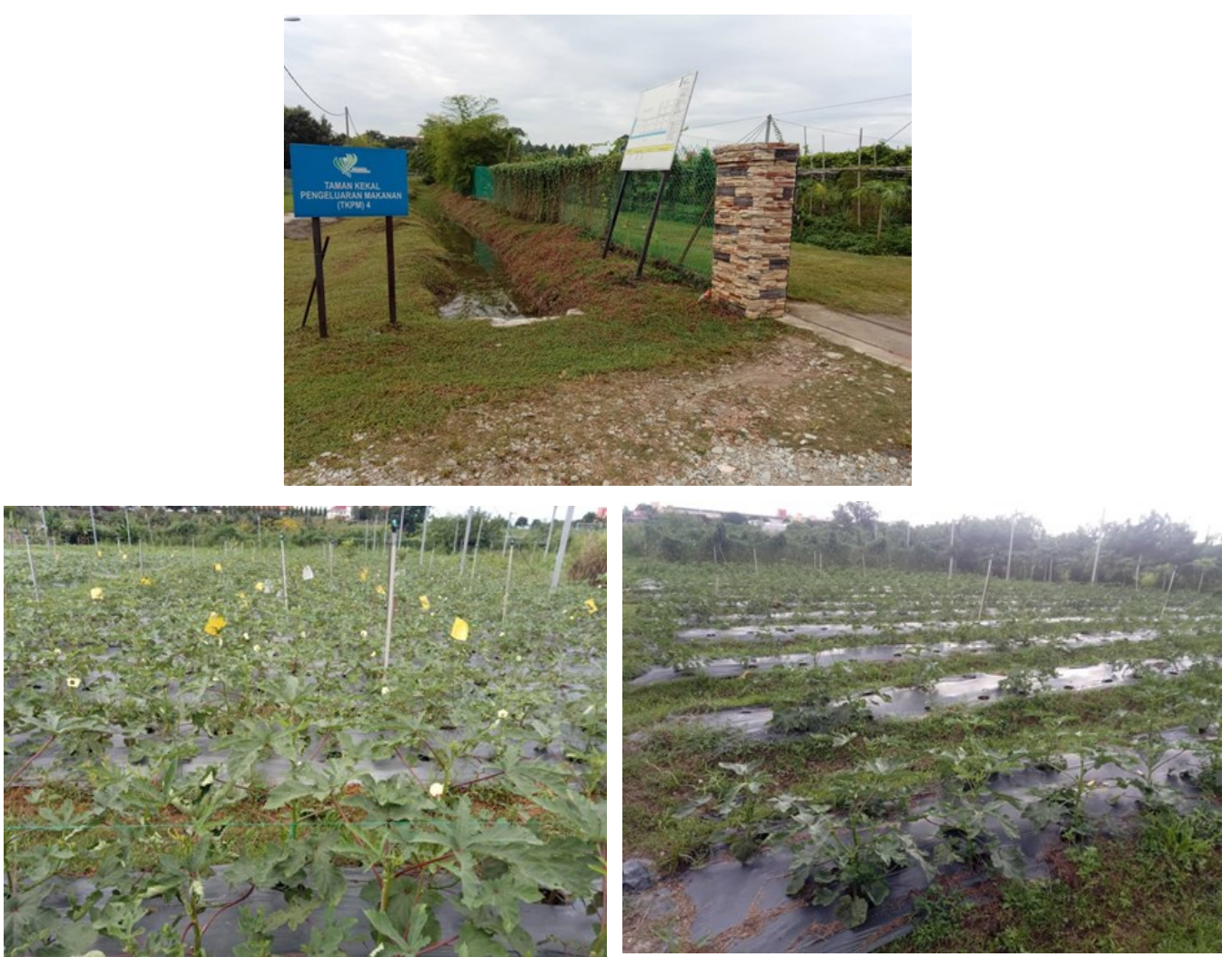

Figure 1. Photos of sampling location, TPKM4 organic farm, Serdang, Selangor 
cephazolin $(30 \mu \mathrm{g})$, ceftazidime $(30 \mu \mathrm{g})$, ciprofloxacin (5 $\mu \mathrm{g})$, erythromycin $(15 \mu \mathrm{g})$, gentamicin $(10 \mu \mathrm{g})$, kanamycin $(30 \mu \mathrm{g})$, penicillin $(10 \mu \mathrm{g})$, nalidixic acid (30 $\mu \mathrm{g})$, streptomycin $(10 \mu \mathrm{g})$, tetracycline $(30 \mu \mathrm{g})$, trimethoprim $(5 \mu \mathrm{g})$ and vancomycin $(30 \mu \mathrm{g})$ (Oxoid, Hamphire, United Kingdom), in accordance with the Clinical and Laboratory Standards Institute guidelines (CLSI, 2012). Multiple antibiotic resistance (MAR) index was calculated as described by Krumperman (1983).

\section{Results and discussion}

In this study, only $2.6 \%(12 / 460)$ of the samples were contaminated with Salmonella spp. For vegetable samples, Salmonella contamination in ladies' fingers $2.5 \%(3 / 120)$ was greater compared to brinjal and cucumber $1.7 \%(2 / 120)$ (Table 1$)$. In accordance with this finding, low prevalence rates of Salmonella spp. in vegetables have been reported in Selangor 4.0\% (3/75) (Kuan et al., 2017) and Perak 3.3\% (6/180) (Saw et al., 2020). The incidence of Salmonella in brinjal, cucumber and ladies' fingers could be due to surface contamination, where the presence of epicuticular wax or uneven surfaces of the vegetable might enhance bacterial adhesion. Some vegetables are grown on the ground which may allow Salmonella present in the soil to cause the contamination. In the present study, we observed $5.0 \%(5 / 100)$ Salmonella in soil samples, which add further support to this vegetable contamination. Perhaps other factors, such as environmental and handling conditions, that would impact the microbial profile of these vegetables.

Out of 12 isolates, 5 serotyped as S. enterica ser. Senftenberg indicating a prevalence rate of $41.67 \%$. Other isolates identified as $S$. enterica ser. Weltevreden $(\mathrm{n}=4)$, and $S$. Corvallis $(\mathrm{n}=3)$. Similarly, the presence of $16.09 \%$ S. enterica ser. Weltevreden $(\mathrm{n}=14)$ and $10.34 \%$ $S$. Corvallis $(\mathrm{n}=9)$ were also found at retail fresh markets in different states of Malaysia including Selangor (Abatcha et al., 2018). On the contrary, a lower (10.1\%) rate of S. enterica ser. Senftenberg incidence has been reported in raw vegetables in the same region (Salleh et al., 2003). Meanwhile, the absence of S. enterica ser. Enteritidis and $S$. enterica ser. Typhimurium in the current study is of interest, as both are well-known serovars to have great concern as foodborne salmonellosis worldwide (Hur et al., 2012). However, a few studies have detected $S$. enterica ser. Enteritidis in Malaysia (Kuan et al., 2017; Abatcha et al., 2018), and S. enterica ser. Typhimurium in Brazil (Sant'Ana et al., 2011), Nigeria (Abakpa et al., 2015) and New Zealand (Wadamori et al., 2016) from different retail vegetables.

We evaluated the resistance profile of Salmonella strains against 14 antimicrobial agents. As shown in Table 2, two antibiotics gentamicin and trimethoprim were found to be $100 \%$ effective. However, a high prevalence of resistance was observed for erythromycin (75.0\%), streptomycin (41.7\%), cephazolin (33.3\%), tetracycline (33.3\%) and ampicillin $(25.0 \%)$. Moreover, all isolates were resistant $(100 \%)$ to penicillin and vancomycin. Similar resistance to antibiotics has been reported for Salmonella spp. from vegetable and soil samples to penicillin and vancomycin (Wadamori et al., 2016). Previous studies also reported a high level of resistance among Salmonella isolates including $S$. enterica ser. Corvallis and $S$. enterica ser. Weltevreden from vegetables to streptomycin (66.6\%), tetracycline (44.3\%) and ampicillin (26.7\%) (Abatcha et al., 2018).

Based on the antibiotic resistance profile (Table 3), all isolates were found to be resistant to at least three different classes of antibiotics. The highest MAR index (0.36) was recorded for ladies' fingers and soil isolates, which found in $S$. Weltevreden with a resistance pattern of KZ-E-P-Te-V. All $S$. Senftenberg and $S$. Corvallis isolates were resistant to four and three antibiotics with MAR index of 0.29 and 0.21 , respectively. These results suggest that MDR (resistant to three or more antimicrobial agents) Salmonella isolates are prevalent in organic farming.

According to certified organic farming practice in Malaysia, there is no use of agricultural chemicals or antimicrobials to produce fresh vegetables. However, other variables, such as the use of contaminated

Table 1. Prevalence of Salmonella in brinjal, cucumber, ladies' fingers and soil

\begin{tabular}{|c|c|c|c|c|c|}
\hline No. & Source & $\begin{array}{c}\text { No. of } \\
\text { samples }\end{array}$ & $\begin{array}{l}\text { No. of positive } \\
\text { samples }\end{array}$ & $\begin{array}{c}\text { Percentage of } \\
\text { positive sample }\end{array}$ & Serotypes \\
\hline 1 & Brinjal & 120 & 2 & $1.7 \%$ & S. enterica ser. Senftenberg $(n=2)$ \\
\hline 2 & Cucumber & 120 & 2 & $1.7 \%$ & $\begin{array}{l}\text { S. enterica ser. Senftenberg }(\mathrm{n}=1) \text { and } S \text {. enterica } \text { ser. } \\
\text { Corvallis }(\mathrm{n}=1)\end{array}$ \\
\hline 3 & Ladies' fingers & 120 & 3 & $2.5 \%$ & $\begin{array}{l}\text { S. enterica } \text { ser. Corvallis }(\mathrm{n}=1) \text { and } S \text {. enterica ser. } \\
\text { Weltevreden }(\mathrm{n}=2)\end{array}$ \\
\hline 4 & Soil & 100 & 5 & $5.0 \%$ & $\begin{array}{l}\text { S. enterica } \text { ser. Corvallis }(n=1), S \text {. enterica ser. } \\
\text { Senftenberg }(n=2) \text { and } S \text {. enterica } \text { ser. Weltevreden } \\
(n=2)\end{array}$ \\
\hline & Total & 460 & 12 & $2.6 \%$ & \\
\hline
\end{tabular}


Table 2. Antimicrobial susceptibility pattern of Salmonella isolates

\begin{tabular}{lcccc}
\hline \multirow{2}{*}{ Antimicrobial agent } & $\begin{array}{c}\text { No. of isolates } \\
\text { tested }\end{array}$ & \multicolumn{2}{c}{ Antibiogram pattern of Salmonella isolates } \\
\cline { 3 - 5 } & 12 & Resistant (\%) & Intermediate (\%) & Sensitive (\%) \\
\hline Amoxicillin (Am30) & 12 & - & $3(25.0)$ & $9(75.0)$ \\
Ampicillin (Amp10) & 12 & $4(25.0)$ & $4(33.3)$ & $5(41.7)$ \\
Cephazolin (KZ30) & 12 & - & - & $8(66.7)$ \\
Ceftazidime (Caz30) & 12 & - & $3(25.0)$ & $9(75.0)$ \\
Ciprofloxacin (Cip5) & 12 & $9(75.0)$ & $3(33.3)$ & $8(66.7)$ \\
Erythromycin (E15) & 12 & - & $3(25.0)$ & - \\
Gentamicin (CN10) & 12 & - & - & $12(100)$ \\
Kanamycin (K30) & 12 & $12(100)$ & $5(41.7)$ & $7(58.3)$ \\
Penicillin (P10) & 12 & - & - & - \\
Nalidixic acid (Na30) & 12 & $5(41.7)$ & $3(25.0)$ & $7(58.3)$ \\
Streptomycin (S10) & 12 & $4(33.3)$ & - & $4(33.3)$ \\
Tetracycline (Te30) & 12 & - & - & $8(66.7)$ \\
Trimethoprim (W5) & 12 & $12(100)$ & - & $12(100)$ \\
Vancomycin (V30) & & & - \\
\hline
\end{tabular}

Table 3. Antibiotic resistance profile and multiple antibiotic resistance (MAR) index of Salmonella isolates

\begin{tabular}{ccllc}
\hline Isolate no. & Source & \multicolumn{1}{c}{ Salmonella serovar } & \multicolumn{1}{c}{ Antibiotic resistance profiles } & MAR index \\
\hline 1 & Brinjal & S. enterica ser. Senftenberg & EPSV & 0.29 \\
2 & Brinjal & S. enterica ser. Senftenberg & EPSV & 0.29 \\
3 & Cucumber & S. enterica ser. Senftenberg & EPSV & 0.29 \\
4 & Cucumber & S. enterica ser. Corvallis & AmpPV & 0.21 \\
5 & Ladies' fingers & S. enterica ser. Corvallis & AmpPV & 0.21 \\
6 & Ladies' fingers & S. enterica ser. Weltevreden & KZEPTeV & 0.36 \\
7 & Ladies' fingers & S. enterica ser. Weltevreden & KZEPTeV & 0.36 \\
8 & Soil & S. enterica ser. Corvallis & AmpPV & 0.21 \\
9 & Soil & S. enterica ser. Senftenberg & EPSV & 0.29 \\
10 & Soil & S. enterica ser. Senftenberg & EPSV & 0.29 \\
11 & Soil & S. enterica ser. Weltevreden & KZEPTeV & 0.36 \\
12 & Soil & S. enterica ser. Weltevreden & KZEPTeV & 0.36 \\
\hline
\end{tabular}

Amp (Ampicillin), KZ (Cephazolin), E (Erythromycin), P (Penicillin), S (Streptomycin), Te (Tetracycline), V (Vancomycin)

irrigation water and incorrect composting process may explain the occurrence of MDR isolates in this study. Indeed, high levels of enteric bacteria, protozoa or helminths are present in contaminated irrigation water, resulting in a high frequency of pathogen detections from harvested fresh produce (Abakpa et al., 2015; Saw et al., 2020). Furthermore, the use of manure from animal faeces, especially from the source of poultry houses, may have significantly contributed to the emergence of MDR strains of Salmonella in vegetable farms (Abatcha et al., 2018). Of particular note, all detected MDR isolates from soil sample could likely be contributed to contamination of vegetable samples in the current study. Therefore, strict surveillance and sustainable agriculture practices at the pre-harvest stage are needed to ensure food safety.

\section{Conclusion}

Our results highlight the potential of organic vegetables may act as reservoirs in harbouring MDR Salmonella. The data provide opportunities for management of antibiotic-resistant Salmonella and risk assessment in the region.

\section{Conflict of interest}

The authors declare no conflict of interest.

\section{Acknowledgments}

This research was supported by the MSU Seed Research Grant (SG-465-0518-HLS) in parts with the Fundamental Research Grant Scheme (FRGS) of the Ministry of Higher Education (MOHE), Malaysia (01-01 -18-2015FR). Appreciation is also expressed to the farmer Mr Cheng Pui Kong of organic farm.

\section{References}

Abakpa, G.O., Umoh, V.J., Ameh, J.B., Yakubu, S.E., Kwaga, J.K.P. and Kamaruzaman, S. (2015). Diversity and antimicrobial resistance of Salmonella enterica isolated from fresh produce and environmental samples. Environmental Nanotechnology, Monitoring and Management, 3, 38 -46. https://doi.org/10.1016/j.enmm.2014.11.004

Abatcha, M.G., Effarizah, M.E. and Rusul, G. (2018). Prevalence, antimicrobial resistance, resistance genes and class 1 integrons of Salmonella serovars in leafy vegetables, chicken carcasses and related processing environments in Malaysian fresh food markets. Food Control, 91, 170-180. https:// doi.org/10.1016/j.foodcont.2018.02.039

Clinical and Laboratory Standards Institute (CLSI) (2012). Performance Standards for Antimicrobial 
disk Susceptibility Tests. Wayne, PA: CLSI.

Food and Drug Administration (FDA) (2020). Salmonella (Salmonellosis). https://www.fda.gov/ food/foodborne-pathogens/salmonella-salmonellosis. Retrieved on 19th June 2020.

Hur, J., Jawale, C. and Lee, J.H. (2012). Antimicrobial resistance of Salmonella isolated from food animals: A review. Food Research International, 45(2), 819830. https://doi.org/10.1016/j.foodres.2011.05.014

ISO (International Organization for Standardization). (2002). Microbiology of Food and Animal Feeding Stuffs-Horizontal Method for the Detection of Salmonella spp. ISO 6579:2002(E). Switzerland.

Krumperman, P.H. (1983). Multiple antibiotic resistance indexing of Escherichia coli to indentify high-risk sources of fecal contamination of foods. Applied and Environmental Microbiology, 46(1), 165-170. https://doi.org/10.1128/AEM.46.1.165-170.1983

Kuan, C.H., Rukayadi, Y., Ahmad, S.H., Wan Mohamed Radzi, C.W.J., Thung, T.Y., Premarathne, J.M.K.J.K., Chang, W.S., Loo, Y.Y., Tan, C.W., Ramzi, O.B., Mohd Fadzil, S.N., Kuan, C.S., Yeo, S.K., Nishibuchi, M. and Radu, S. (2017). Comparison of the microbiological quality and safety between conventional and organic vegetables sold in Malaysia. Frontiers in Microbiology, 8, 1433. https://doi.org/10.3389/fmicb.2017.01433

Saira Banu, M.R., Humairak, S., Zakiah, M.D. and Adila, Z.A. (2019). Retrospective study on persistent Salmonella serotypes in meat samples tested in the veterinary public health section, Regional of Veterinary Laboratory Bukit Tengah, Penang. Malaysian Journal of Veterinary Research 10(1), 3442

Salleh, N.A., Rusul, G., Hassan, Z., Reezal, A., Isa, S.H., Nishibuchi, M. and Radu, S. (2003). Incidence of Salmonella spp. in raw vegetables in Selangor, Malaysia. Food Control, 14(7), 475-479. https:// doi.org/10.1016/S0956-7135(02)00105-6

Sant'Ana, A.S., Landgraf, M., Destro, M.T. and Franco, B.D.G.M. (2011). Prevalence and counts of Salmonella spp. in minimally processed vegetables in São Paulo, Brazil. Foods Microbiology, 28(6), 1235-1237. https://doi.org/10.1016/j.fm.2011.04.002

Saw, S.H., Mak, J.L., Tan, M.H., Teo, S.T., Tan, T.Y., Cheow, M.Y.K., Ong, C.A., Chen, S.N., Yeo, S.K., Kuan, C.S., Son, R., New, C.Y., Phuah, E.T., Thung, T.Y. and Kuan, C.H. (2020). Detection and quantification of Salmonella in fresh vegetables in Perak, Malaysia. Food Research, 4(2), 441-448. https://doi.org/10.26656/fr.2017.4(2).316
Wadamori, Y., Fam, J., Hussain, M.A. and Gooneratne, R. (2016). Microbiological risk assessment and antibiotic resistance profiling of fresh produce from different soil enrichment systems: A preliminary study. Cogent Food and Agriculture, 2(1), 1274281. https://doi.org/10.1080/23311932.2016.1274281 